\title{
Predictive factors in early onset schizophrenia
}

\author{
MAGDALENA BUDISTEANU ${ }^{1-3^{*}}$, EMANUELA ANDREI ${ }^{1}$, FLORENTINA LINCA $^{1}$, DIANA STEFANIA HULEA ${ }^{4}$ \\ ALEXANDRA CATALINA VELICU ${ }^{4}$, ILINCA MIHAILESCU ${ }^{1}$, SORIN RIGA $^{1}$, AURORA ARGHIR ${ }^{2}$, \\ SORINA MIHAELA PAPUC ${ }^{2}$, CARMEN ADELLA SIRBU $^{5}$, MARIAN MITRICA $^{6^{*}}$, ANY DOCU-AXELERAD ${ }^{7,8}$, \\ MINERVA CLAUDIA GHINESCU ${ }^{5 *}$, IULIANA DOBRESCU ${ }^{1,4}$ and FLORINA RAD ${ }^{1,4}$ \\ ${ }^{1}$ Psychiatry Research Laboratory, 'Prof. Dr. Alexandru Obregia’ Clinical Hospital of Psychiatry, 041914 Bucharest; \\ ${ }^{2}$ Medical Genetics Laboratory, 'Victor Babes' National Institute of Pathology, 050096 Bucharest; \\ ${ }^{3}$ Department of Preclinical Disciplines, Faculty of Medicine, 'Titu Maiorescu' University, 031593 Bucharest; \\ ${ }^{4}$ Department of Clinical Neuroscience, 'Carol Davila' University of Medicine and Pharmacy, 050474 Bucharest; \\ ${ }^{5}$ Department of Medical-Surgical and Prophylactical Disciplines, Faculty of Medicine, 'Titu Maiorescu' University, \\ 031593 Bucharest; ' ${ }^{2}$ Department of Neurosurgery, 'Dr. Carol Davila Central Military Emergency University Hospital', \\ 010242 Bucharest; ${ }^{7}$ Department of Neurology, Faculty of Medicine, 'Ovidius' University of Constanta; \\ ${ }^{8}$ Department of Neurology, Clinical Emergency Hospital 'St. Apostol Andrei', \\ ‘Ovidius’ University of Constanta, 900527 Constanta, Romania
}

Received August 28, 2020; Accepted September 29, 2020

DOI: $10.3892 /$ etm. 2020.9340

\begin{abstract}
Schizophrenia is a neurodevelopmental disorder, characterized by impairment in reasoning, affectivity and social relationships. Although the diagnosis of schizophrenia in children and adolescents has been challenged for many years, at present childhood-onset schizophrenia is considered and accepted as a clinical and biological continuum with the adult-onset disorder. The present study aimed to evaluate the influence of biological (psychiatric family history, perinatal factors), and socio-demographic factors (area of residence, gender) on the age at onset and severity of symptomatology in children and adolescent with schizophrenia. The data were collected from 2016 to 2019 and included 148 children and adolescents with schizophrenia. Data were analysed with statistical software (IBM SPSS 22, JASP and JAMOVI, Linear Regression Model, $\chi^{2}$ tests, t-test, U-test). A positive familial history for psychiatric diseases was an important risk factor both for an early onset and for the severity of symptoms. Urbanicity was another studied risk factor, $61 \%$ of patients being from urban areas; no statistically significant correlations
\end{abstract}

Correspondence to: Dr Carmen Adella Sirbu, Department of Medical-Surgical and Prophylactical Disciplines, Faculty of Medicine, 'Titu Maiorescu' University, 67A Gheorghe Petraşcu Street, 031593 Bucharest, Romania

E-mail: sircar13@yahoo.com

*Contributed equally

Key words: schizophrenia, children, adolescents, biological factors, socio-demographic factors between urbanicity and age at onset and severity of symptoms were identified. There was no statistically significant gender difference in terms of age at onset and severity of symptoms. Moreover, no statistically significant correlations were found between perinatal factors and age at onset and severity of symptoms. Positive psychiatric family history showed a statistically significant influence on age at onset and symptoms severity in children and adolescent schizophrenia; no statistical significant impact on the aforementioned schizophrenia aspects was observed for urbanicity, gender or perinatal factors.

\section{Introduction}

Schizophrenia is described as one of the most mysterious and, at the same time, one of the most crippling of psychiatric disorders (1). Though uncommon before 10 years of age, the incidence of schizophrenia increases progressively from adolescence to adulthood. Early-onset schizophrenia (EOS) that is diagnosed in childhood and adolescence is currently viewed as a clinical and biological continuum with the adult-specific disorder (2). EOS refers to the onset of symptoms by 18 years of age, while a debut before 13 years of age is defined as childhood-onset schizophrenia (COS) or very early onset schizophrenia (VEOS) (3). The prevalence of schizophrenia at a worldwide level is appreciated at $0.33-0.75 \%(4,5)$. The prevalence of EOS has not been adequately studied so far, as, for years EOS had not been taken into consideration as a distinct disorder. Some authors estimate a general prevalence of clinically defined EOS to be $<4 \%$ (2). EOS tends to occur more often in male individuals. As age increases, this ratio tends to even out (3).

It has been proven that no single set of causes can be pinned down as an underlying mechanism for this condition, schizophrenia being considered a heterogeneous disorder 
with multiple aetiologies. Current evidence suggests that a model with multiple factors interfering with normal neurodevelopmental milestones best explain the development of schizophrenia, the complex interplay between genetic and epigenetic, environmental and social factors contributing to this outcome $(3,6,7)$. Schizophrenia is highly heritable $(80 \%)$, though in many patients the illness is sporadic with no family history of psychosis. Existing genetic data suggest that multiple genetic variants of varying frequencies (common to rare variants) contribute (2). Numerous environmental factors have been hypothesized to contribute to the development of schizophrenia, including paternal age, in utero exposure to maternal malnutrition or infectious agents, obstetric complications, cannabis use during adolescence, and immigration (3).

A family history of psychiatric conditions is considered to be the strongest risk factor for schizophrenia among first-degree relatives (8). Most of the related studies demonstrated that positive family history is associated with an earlier age at onset, severity of symptoms and the duration of untreated illness (9-11). Besides the positive history of schizophrenia in first-degree relatives, Mortensen et al (12) in 1999 showed that a significant portion of the relative risks is accounted by the place of birth, as well as the season of birth.

A series of pre- and perinatal risk factors have been identified, significantly associated with schizophrenia including complications of pregnancy (bleeding, diabetes, pre-eclampsia), abnormal intrauterine development (low birth weight, congenital malformations, reduced head circumference) and complications of delivery (uterine atony, asphyxia, emergency Caesarean section) (13). Other significant associations have been observed between schizophrenia and gestational age less than 37 weeks, the use of resuscitation or an incubator, while birth weight less than 2,500 $\mathrm{g}$ or forceps delivery represents factors of borderline significance (14).

Clinical observation shows that men and women have different outcome of the schizophrenia age at onset, symptoms, and disease severity. The disease onset occurs at a significantly earlier age in male patients in comparison to female patients. While male patients show higher susceptibility to negative symptoms, cognitive impairment, and co-morbid substance abuse, female patients often present with more mood disturbance and depressive symptoms as well as affective symptoms (15). Other factors which proved to have an impact on age at onset and symptoms severity were a positive familial history for psychiatric diseases and a delayed psychomotor development (16-20); urbanicity and perinatal events were also studied as important risk factors for EOS (21). However, the influence of the aforementioned factors on age at onset and symptoms severity in children and adolescents with schizophrenia is less explored.

This study presents our investigation on the influence of familial and personal medical history and socio-demographic factors on the age at onset and severity of schizophrenia in children and adolescents.

\section{Materials and methods}

A retrospective study was performed in the Department of Child and Adolescent Psychiatry of 'Prof. Dr. Alexandru
Obregia' Clinical Hospital of Psychiatry (Bucharest, Romania), a tertiary center in Bucharest, and included children and adolescents admitted for the first time between January 2016 and June 2019, most of them originating from the South and South-Eastern regions of Romania, with diagnosis of schizophrenia. The diagnosis was established according to ICD-10 criteria (22). All data were collected by review of hospital records from the first admission to the hospital, at diagnosis. Age, gender and area of residence were collected from the demographic section of each patient records; the clinical findings at diagnosis, most importantly the types and severity of symptoms, were also collected from the diagnostic medical records. Personal and familial history collected from patient records were obtained by parents interviews. A special emphasis was placed on:

i) existence of a familial history positive for psychiatric disorders (family study performed by diagnostic interviews conducted with the patient's parents in most cases, ocasionally with other family members; for 1st degree relative we included parents, brothers, sisters, for 2nd degree relatives, aunts, uncles, grandparents, or half-siblings of an individual);

ii) presence of perinatal factors was defined as follows: pathological events during pregnancy and/or at birth (birth asphyxia and/or hypoxia, difficult postnatal adaptation); type of birth (natural birth or caesarean section); birth weight (we used a threshold of 2,500 $\mathrm{g}$ to define a lower birth weight for babies born full term);

iii) a personal history positive for psychomotor developmental delay;

iv) a personal history positive for other psychiatric conditions (hyperkinesia, behaviour problems, etc.).

These data were translated into a set of binary variables. The place of residence was coded using a two-category classification according to the degree of urbanization: urban areas (population $>5,000$, according to national legislation) and rural areas.

According to ICD-10, positive symptoms include hallucinations, delusions, disorganised thinking, while negative symptoms encompass marked apathy, paucity of speech, and blunting or incongruity of emotional responses. The severity of symptoms was recorded at diagnosis, and coded using symptom severity scale of the DSM-5 for schizophrenia: 0 , not present; 1, equivocal; 2, present, but mild, 3, present and moderate; 4, present and severe (23). All these variables are continuous.

All patients were evaluated by general clinical examination, psychiatric, neurological and psychological evaluation. Supplementary investigations included blood tests, electroencephalogram and neuroimagistic studies (CT, MRI).

Statistical analysis of data was performed using IBM SPSS Statistics Version 22 (IBM Corp for Windows), JASP and JAMOVI software. Linear regression models were applied to verify the existence of relationships between variables such as severity of symptoms or age of onset, and biological and demographic factors. The t-test and U-test were applied to evaluate the differences in severity of symptoms based on criteria such as residence, gender and type of birth. Ethical approval for the study was obtained from the local research ethics committee. A written informed consent was signed by the parents or legal guardian for all patients included in this study. 
Table I. Demographic characteristics of the patients.

\begin{tabular}{|c|c|c|}
\hline Characteristics & Frequency & Percent \\
\hline \multicolumn{3}{|l|}{ Heredocolateral background } \\
\hline Without psychiatric history & 71 & 48.0 \\
\hline Psychiatric history & 77 & 52.0 \\
\hline \multicolumn{3}{|l|}{ Psychiatric history } \\
\hline Family history of schizophrenia (relatives of 1st and 2nd degree) & 53 & 69.0 \\
\hline Family history of major depression (relatives of 1st degree) & 12 & 16.0 \\
\hline Family history of ASD (relatives of 1st degree) & 5 & 6.5 \\
\hline Family history of ADHD (relatives of 1st degree) & 5 & 6.5 \\
\hline Family history of substance use disorders, alcoholism (relatives of 1st degree) & 2 & 3.0 \\
\hline \multicolumn{3}{|l|}{ Peri/postnatal suffering } \\
\hline Yes & 46 & 21.0 \\
\hline No & 102 & 69.0 \\
\hline \multicolumn{3}{|l|}{ Residence } \\
\hline Rural area & 57 & 39.0 \\
\hline Urban area & 91 & 61.0 \\
\hline \multicolumn{3}{|l|}{ Sex } \\
\hline Male & 92 & 62.2 \\
\hline Female & 56 & 37.8 \\
\hline \multicolumn{3}{|l|}{ Psychomotor disorders } \\
\hline Yes & 51 & 34.45 \\
\hline No & 97 & 66.55 \\
\hline
\end{tabular}

ASD, autism spectrum disorder; ADHD, attention-deficit hyperactivity disorder.

\section{Results}

The study included 148 patients (92 boys and 56 girls). The age range was 11 to 17 years [mean age $=15.71$ years, and a standard deviation $(\mathrm{SD})=1.39$ ]. Auditory and/or visual hallucinations were the main manifestations in most of the patients $(n=136)$; delusions and hetero-aggressively, associated with psychomotor hyperkinesia and a bizarre behaviour were noted in 12 patients. In 21 patients the onset of psychiatric manifestations was associated with drug abuse (cocaine and marijuana).

Ninety-one patients $(61 \%)$ lived in an urban area, and 57 patients $(39 \%)$ originated from a rural area. There were insignificant differences depending on the place of residence in terms of age at onset of symptoms and severity of the symptomatology $(\mathrm{P}>0.05)$. However, patients from urban area had higher averages in terms of symptom severity and age at onset of symptoms.

A psychiatric history in the family was noted in 77 participants (52\%): 53 have a family history of schizophrenia (relatives of 1 st and 2nd degree), 12 have a family history of major depression (relatives of 1st degree), 5 have a family history of autism spectrum disorder (ASD) (relatives of 1st degree), 5 have a family history of attention-deficit hyperactivity disorder (ADHD) (relatives of 1st degree), and 2 have a family history of substance use disorders, alcoholism (relatives of 1st degree). There is a statistically significant positive relationship between age at onset and the severity of the symptoms and a positive familial history for psychiatric diseases $(\mathrm{P}<0.05)$. We also observed a statistically significant positive relation- ship between the severity of the symptoms and age at onset of the symptoms $(\mathrm{P}<0.05)$. Furthermore, a significant association between severity of symptoms and a positive familial history, adjusting for age of onset was observed $(\mathrm{P}<0.05)$.

A delayed psychomotor development was noted in 51 cases; 8 patients had a previous diagnosis of Asperger syndrome. No statistically significant correlations between psychomotor development and severity of symptoms or age at onset were noted $(\mathrm{P}>0.05)$.

Regarding the differences depending on gender in terms of age at onset of the symptoms and severity of the symptomatology, although the boys had a higher severity of symptoms, the results were not statistically significant $(M=0.46$ severity of symptoms, $\mathrm{M}=0.42$ age at onset of the symptoms, $\mathrm{P}>0.05$ ).

With regard to the role of perinatal events on the severity of symptomatology, in our cohort, 46 patients had complications at birth, and 46 subjects were born by caesarean section; these data were not statistically significant $(\mathrm{P}>0.05)$. Also, regarding the differences depending on birth weight in terms of age at onset and severity of the symptomatology, the data were not statistically significant $(\mathrm{P}>0.05)$.

In summary, a statistically significant influence both on the age of onset and on the severity of symptoms was observed for positive psychiatric family history. All the other factors studied in relation to the age of onset and severity of symptoms were not found to be statistically significant (Table I). However, our data points to other interesting associations, such as the influence of area of residence. 


\section{Discussion}

Schizophrenia is a rare condition in children and teenagers, both genetic and environmental factors being studied as risk factors. Our study aimed to investigate some of the most frequent risk factors for EOS, namely family history positive for schizophrenia or other psychiatric conditions, gender, place of residence, and perinatal factors, and their impact on age at onset and symptoms severity. Familial history positive for psychiatric diseases was recurrent in our study group, $52 \%$ of the patients having relatives of first or second degree with different psychiatric conditions, most of them $(67 \%)$ represented by schizophrenia. Our statistical analysis showed that the presence of psychiatric disorders correlates with a more severe clinical picture and early onset.

Similar data were reported also by other authors. Thus, Chou et al (16) found a prevalence of schizophrenia of 6.3-fold higher in subjects with a first-degree relative with schizophrenia and 2.4-fold higher in persons with a second-degree relative with schizophrenia, comparing with general population. Nicolson et al (17) showed that patients with EOS have a greater familial risk than those with adult onset, and Hollis (18) found that $50 \%$ of subjects with EOS had a first-degree relative with psychosis. The familial history of psychiatric disorders correlated also with the severity of the symptoms; the same correlation was observed also for the age of the onset of the symptoms. This finding was reported by Mäki et al (19) in a previous study, which revealed that a positive familial history for psychiatric diseases was a strong predictor for the severity of schizophrenia symptoms.

Previous studies showed an association between development of schizophrenia and a delayed psychomotor development in the first years of life. This association could be explained by intervention of some genetic factors and/or of perinatal events, sometimes in combination with negative environmental factors (such as cognitive and/or affective deprivation, child neglect) (20). Also, some authors reported that up to $27 \%$ of subjects with adult-onset schizophrenia had a history of autism as part of an impaired psychomotor development, considered as a premorbid phenotype for COS (24). In our cohort, only 51 patients had psychomotor delay, out of these 8 patients having a previous diagnosis of Asperger syndrome; these data were not statistically significant. Motor abnormalities have long been observed in patients with schizophrenia, and antedate the use of neuroleptics. It was demonstrated that simple movements are stiff, slow, forced and include abnormalities in motor coordination (25).

Regarding the place of residence, most of the patients included in our study (61\%) were located in an urban area. These data are in concordance with previous studies which showed that the rate of schizophrenia in urban areas is around double the rate of the rate in rural areas, suggesting that the environmental factors in the urban areas act on the pre-existing genetic background for psychosis (26). It is considered that this higher risk would be related to the correlation of some adverse social situations, including population density, social fragmentation and deprivation, with different environmental exposures at the individual level, such as drug use, discrimination and exclusion (27). Also, it has been suggested that repeated exposures to different risk factors such as infection and toxins during child development, which are more often present in urban area, raise the risk of psychosis (21). However, in terms of age at onset or symptoms severity, our results did not reach the threshold for statistical significance. Comparison with previous data is difficult, as to our knowledge, the influence of urbanicity on age at onset or severity of symptoms in children and adolescent with schizophrenia has not been studied before.

In our study the number of male patients was higher than that of females (92 vs. 56), but there were no statistically significant gender differences in terms of age at onset and severity of the symptoms. However, boys had a higher severity of symptoms than girls. The epidemiological studies on schizophrenia showed that there are no gender differences, but males have an earlier onset of the symptoms and a higher prevalence of negative symptoms than females (28).

Regarding the perinatal factors (perinatal hypoxia and/or ischemia), in our cohort these were noted in 46 patients (31\%); there were no statistically significant correlations between obstetric complications, type of birth and birth weight, and age at onset of symptoms and the severity of the symptomatology. Previous studies showed that subjects with schizophrenia experienced more often different obstetric complications, such as hypoxia at birth, compared to their unaffected siblings. Moreover, Jenkins (21) found brain anomalies (such as reduced grey matter and ventricular enlargement) in schizophrenia patients with perinatal hypoxia. Other authors consider that obstetric complications represent a non-specific risk factor for schizophrenia, of small effect, and rather the interaction between genotype and environmental factors (including pre- and perinatal events), would lead to this complex condition (29). Previous studies showed that a lower birth weight has been associated with a higher risk of schizophrenia, often in association with a smaller occipital frontal circumference; this growth retardation would be related to some genetic factors, but a clear correlation with schizophrenia from the point of view of pathogenesis could not be established (29).

Our study has several limitations. Although, selection bias is probably unlikely, as patients from South and South-Eastern regions of Romania are routinely referred to our center, some patients might have been addressed to other centers in Romania. Also, we have collected the family history data by interview only, as very few families can provide medical documentation on family members.

Schizophrenia is a devastating condition, particularly when presenting in childhood or adolescence. An increased evidence base approach concerning the clinical presentation and course of psychosis in childhood and adolescence is the first step toward identifying improved strategies for early detection and development of more operational treatment guidelines. Identification of the risk factors for an early onset of schizophrenia and for prediction of the severity of the symptoms will contribute to a better management of this condition. A familial history positive for psychiatric conditions proved to be an important risk factor both for an early onset of the disease and for severity of the symptoms, in our study group. Therefore, children from the families with psychiatric conditions (mainly schizophrenia or other psychosis) should be more carefully followed for psychi- 
atric symptoms in the primary care setting; these families should, also, benefit of a proper genetic counselling regarding the recurrence risk of the disease. Other risk factors such as urbanicity, gender and pre- and/or perinatal factors, such as obstetrical complications (birth hypoxia/asphyxia) and low birth weight, were not statistically significant for age at onset or symptoms severity. Overall, our study brings additional data regarding factors predictive of early onset and severity of symptoms in children and adolescents, a schizophrenia population group less intensely studied, so far.

\section{Acknowledgements}

We would like to thank Mrs Iuliana Ciocanea-Teodorescu for her help during the revision of the manuscript.

\section{Funding}

This work was supported partially by a grant of Romanian National Authority for Scientific Research and Innovation CCCDI-UEFISCDI project number COFUND-ERANET NEURON SYNSCHIZ 6/2018 and by Ministry of Education and Research in Romania, under Program 1-The Improvement of the National System of Research and Development, Subprogram 1.2-Institutional Excellence-Projects of Excellence Funding in RDI, contract no. 7PFE/16.10.2018.

\section{Availability of data and materials}

The data and material used and/or analyzed during the current study are available from the corresponding author on reasonable request.

\section{Authors' contributions}

MB, CAS, MM and MCG was involved in the conception of the study. EA, FL, ADA and ID participated in the design of the study. DSH, ACV, IM, SR, AA, SMP and FR have substantial contribution to the analysis and data interpretation. FR, ID, MCG, ADA, MM, CAS and SMP drafted the work. AA, SR, IM, ACV, DSH, FL, EA and MB critically reviewed the literature data. All authors approved the manuscript and agree to be accountable for all aspects of the research in ensuring that the accuracy or integrity of any part of the work are appropriately investigated and resolved.

\section{Ethics approval and consent to participate}

This study was approved by the Ethics Committee of 'Prof. Dr. Alexandru Obregia' Clinical Hospital of Psychiatry, (Bucharest, Romania).

\section{Patient consent for publication}

Written informed consent was obtained from the legal guardian of each patient.

\section{Competing interests}

The authors declare that they have no competing interests.

\section{References}

1. Tamminga CA: Schizophrenia and other psychotic disorders. In: Kaplan \& Sadock's comprehensive textbook of psychiatry. Sadock BJ, Sadock VA, Ruiz P and Kaplan HI (eds). 9th edition, Wolters Kluwer Health/Lippincott Williams \& Wilkins, Philadelphia, PA, pp1432-1628, 2009.

2. Hollis C and Palaniyappan L: Schizophrenia and psychosis. In: Rutter's Child and Adolescent Psychiatry. Thapar A, Pine DS, Leckman JF, Scott S, Snowling MJ and Taylor E (eds). 6th edition. John Wiley \& Sons Ltd., Hoboken, NJ, pp774-794, 2015.

3. McClellan J and Stock S; American Academy of Child and Adolescent Psychiatry (AACAP) Committee on Quality Issues (CQI): Practice parameter for the assessment and treatment of children and adolescents with schizophrenia. J Am Acad Child Adolesc Psychiatry 52: 976-990, 2013.

4. Saha S, Chant D, Welham J and McGrath J: A systematic review of the prevalence of schizophrenia. PLoS Med 2: e141, 2005.

5. Moreno-Küstner B, Martín C and Pastor L: Prevalence of psychotic disorders and its association with methodological issues. A systematic review and meta-analyses. PLoS One 13: e0195687, 2018.

6. Davis J, Eyre H, Jacka FN, Dodd S, Dean O, McEwen S, Debnath M, McGrath J, Maes M, Amminger P, et al: A review of vulnerability and risks for schizophrenia: Beyond the two hit hypothesis. Neurosci Biobehav Rev 65: 185-194, 2016.

7. Murray RM, Bhavsar V, Tripoli G and Howes O: 30 Years on: How the neurodevelopmental hypothesis of schizophrenia morphed into the developmental risk factor model of psychosis. Schizophr Bull 43: 1190-1196, 2017.

8. Mortensen PB, Pedersen MG and Pedersen CB: Psychiatric family history and schizophrenia risk in Denmark: Which mental disorders are relevant? Psychol Med 40: 201-210, 2010.

9. Ritsner MS, Ratner Y, Gibel A and Weizman R: Familiality in a five-factor model of schizophrenia psychopathology: Findings from a 16-month follow-up study. Psychiatry Res 136: 173-179, 2005.

10. Ritsner MS, Ratner Y, Gibel A and Weizman R: Positive family history is associated with persistent elevated emotional distress in schizophrenia: Evidence from a 16-month follow-up study. Psychiatry Res 153: 217-223, 2007.

11. Esterberg $M$ and Compton M: Family history of psychosis negatively impacts age at onset, negative symptoms, and duration of untreated illness and psychosis in first-episode psychosis patients. Psychiatry Res 197: 23-28, 2012.

12. Mortensen PB, Pedersen CB, Westergaard T, Wohlfahrt J, Ewald H, Mors O, Andersen PK and Melbye M: Effects of family history and place and season of birth on the risk of schizophrenia. N Engl J Med 340: 603-608, 1999.

13. Cannon M, Jones PB and Murray RM: Obstetric complications and schizophrenia: Historical and meta-analytic review. Am J Psychiatry 159: 1080-1092, 2002.

14. Geddes JR, Verdoux H, Takei N, Lawrie SM, Bovet P, Eagles JM, Heun R, McCreadie RG, McNeil TF, O'Callaghan E, et al: Schizophrenia and complications of pregnancy and labor: An individual patient data meta-analysis. Schizophr Bull 25: 413-423, 1999.

15. Nawka A, Kalisova L, Raboch J, Giacco D, Cihal L, Onchev G, Karastergiou A, Solomon Z, Fiorillo A, Del Vecchio V, et al: Gender differences in coerced patients with schizophrenia. BMC Psychiatry 13: 257, 2013.

16. Chou IJ, Kuo CF, Huang YS, Grainge MJ, Valdes AM, See LC, Yu KH, Luo SF, Huang LS, Tseng WY, et al: Familial aggregation and heritability of schizophrenia and co-aggregation of psychiatric illnesses in affected families. Schizophr Bull 43: 1070-1078, 2017.

17. Nicolson R, Brookner FB, Lenane M, Gochman P, Ingraham LJ, Egan MF, Kendler KS, Pickar D, Weinberger DR and Rapoport JL: Parental schizophrenia spectrum disorders in childhood-onset and adult-onset schizophrenia. Am J Psychiatry 160: 490-495, 2003.

18. Hollis C: A study of the course and adult outcomes of child and adolescent onset psychoses (unpublished $\mathrm{PhD}$ thesis). University of London, 1999.

19. Mäki P, Veijola J, Jones PB, Murray GK, Koponen H, Tienari P, Miettunen J, Tanskanen P, Wahlberg KE, Koskinen J, et al: Predictors of schizophrenia - a review. Br Med Bull 74: 1-15, 2005.

20. Sørensena HJ, Mortensena EL, Schiffmand J, Reinischa JM, Maedaf J and Mednick SA: Early developmental milestones and risk of schizophrenia: A 45-year follow-up of the Copenhagen perinatal cohort. Schizophr Res 118: 41-47, 2010. 
21. Jenkins TA: Perinatal complications and schizophrenia: Involvement of the immune system. Front Neurosci 7: 110, 2013.

22. World Health Organization (WHO): International statistical classification of diseases and related health problems. 10th revision. 5th edition. WHO, Geneva, pp293-299, 2016.

23. American Psychiatric Association: Diagnostic and statistical manual of mental disorders (DSM-5). 5th edition. American Psychiatric Association Publishing, Washington, DC, 2016.

24. Driver DI, Gogtay N and Rapoport JL: Childhood onset schizophrenia and early onset schizophrenia spectrum disorders. Child Adolesc Psychiatr Clin N Am 22: 539-555, 2013.

25. Jaspers K: General psychopathology. University of Chicago Press, Chicago, 1965.
26. Krabbendam L and van Os J: Schizophrenia and urbanicity: A major environmental influence - conditional on genetic risk. Schizophr Bull 31: 795-799, 2005.

27. Heinz A, Desemo L and Reininghaus U: Urbanicity, social adversity and psychosis. World Psychiatry 12: 187-197, 2013.

28. Ochoa S, Usall J, Cobo J, Labad X and Kulkarni J: Gender differences in schizophrenia and first-episode psychosis: A comprehensive literature review. Schizophr Res Treatment 2012: 916198, 2012.

29. Clarke MC, Harley M and Cannon M: The role of obstetric events in schizophrenia. Schizopr Bull 32: 3-8, 2006. 